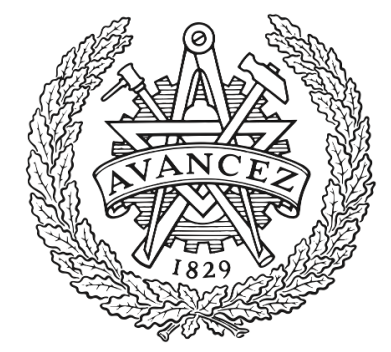

CHALMERS

UNIVERSITY OF TECHNOLOGY

\title{
Network Performance Trade-Off in Modular Data Centers With Optical Spatial Division Multiplexing
}

Downloaded from: https://research.chalmers.se, 2023-04-26 15:17 UTC

Citation for the original published paper (version of record):

Yan, L., Fiorani, M., Muhammad, A. et al (2018). Network Performance Trade-Off in Modular Data Centers With Optical Spatial Division Multiplexing. Journal of Optical Communications and Networking, 10(9): 796-808. http://dx.doi.org/10.1364/JOCN.10.000796

N.B. When citing this work, cite the original published paper. 


\title{
Network Performance Trade-Off in Modular Data Centers With Optical Spatial Division Multiplexing
}

\author{
Li Yan, Matteo Fiorani, Ajmal Muhammad, Massimo Tornatore, \\ Erik Agrell, and Lena Wosinska
}

\begin{abstract}
The modular design based on spatial division multiplexing switches is recently proposed to improve the capacity and reduce the cabling complexity of data center networks. However, due to the coexistence of mice and elephant flows, in modular data center networks a trade-off between the blocking probability and total throughput arises. In fact, blocking elephant flows would lead to a relevant penalty on the throughput, but a small penalty on the blocking probability. In this paper, we investigate the relation between the blocking and throughput in modular data center networks based on optical spatial division multiplexing. The two metrics are combined linearly by a weight factor that prioritizes them relatively. Both mixed integer linear programming formulations and close-to-optimal heuristics based on problem decomposition are proposed for three different spatial division multiplexing switching schemes. Simulation results demonstrate that a carefully chosen weight factor is necessary to achieve a proper balance between the blocking probability and throughput for all the schemes.
\end{abstract}

Index Terms-Spatial division multiplexing, data centers, resource allocation.

\section{INTRODUCTION}

Driven by the increasing adoption of cloud services, the demand for large data centers (DCs) is growing rapidly. The modular DC [1] based on prefabricated stand-alone modules, referred to as PODs, is considered as an effective solution to build and maintain large DC facilities. Each POD typically holds several hundred servers, which are equipped with network interface cards operating at the data rate of $10 \mathrm{Gbps}$ or higher [2]. Due to the massive amount of required bandwidth, it is a significant challenge to interconnect hundreds of such PODs to form a modular DC.

Optical spatial division multiplexing (SDM) enabled by multicore, multimode, or multielement fibers has been recently proposed as a cost-effective and energy-efficient solution to boost the network capacity of DC networks [3]. Moreover, SDM can be combined with wavelength division multiplexing (WDM) to achieve even higher capacity [4], [5]. Depending on whether optical signals on different spectral and spatial elements are coupled to form superchannels, several SDM switching schemes with different levels of flexibility and complexity have been investigated in [4]-[6].

Manuscript received XXX. XX, 2017; revised XXX. Xx, 2017; accepted XXX. xx, 2017; published XXX. Xx, 2017.

L. Yan and E. Agrell are with Chalmers University of Technology, Gothenburg, Sweden (e-mail: lyaa@chalmers.se).

M. Fiorani, A. Muhammad, and L. Wosinska are with KTH Royal Institute of Technology, School of ICT, Stockholm, Sweden.

M. Tornatore is with Politecnico di Milano, Milano, Italy.
To fully exploit the advantages of SDM-based modular DC networks, it is of great importance to develop high performance resource allocation algorithms. Several spectral and spatial superchannel allocation policies aiming at minimizing the blocking probability of backbone networks are studied and compared in [7]-[9]. An integer linear programming formulation as well as a scalable heuristic algorithm are proposed in [10] to minimize the resource usage and, thus, maximizing the traffic throughput of backbone SDM networks. Simple first-fit (FF) resource allocation strategies are proposed in [5], [6] for SDM-based modular DCs to effectively evaluate the blocking probabilities of different SDM switching schemes. In [11]-[13] the traffic throughput of nonlinear backbone networks is maximized by optimizing the transmitter power and route of light paths. Other resource allocation strategies considering various physical layer impairments in multicore fibers while optimizing either the blocking probability or throughput can be found in [14], [15].

The algorithms above can be classified into two main categories based on their optimization objective: (i) the throughput maximization ${ }^{1}$, which aims at provisioning the highest amount of traffic in the network [10]-[13], [15] and (ii) the blocking probability minimization, which aims at minimizing the number of connections that cannot be established [5][9], [14]. These two objectives are normally closely related as an increased number of established connections leads to a higher throughput. However, this is not necessarily the case in modular DC networks, where the data rates of traffic demands are highly unbalanced. Previous studies [16]-[19] have revealed that the majority of flows within the DC networks have low data rates, yet the majority of throughput belongs to a few elephant flows. This unbalance becomes more severe when the traffic demands are aggregated in the modular DCs, which makes the contrast between different flow classes even stronger. Consequently, targeting the minimization of the blocking probability may incur high blocking of bandwidthintensive elephant connections and hence reduce the network throughput. On the other hand, maximizing the throughput may lead to blocking of an excessive number of mice flows. Therefore, a balance between the blocking probability and throughput should be sought to guarantee fairness and efficiency in DC networks.

In [20], we identified and analyzed the trade-off behavior between the blocking probability and throughput by using

\footnotetext{
${ }^{1}$ The minimization of the blocking probability measured in bandwidth is equivalent to the throughput maximization in the offline resource allocation problem and, thus, will not be explicitly considered in this paper.
} 


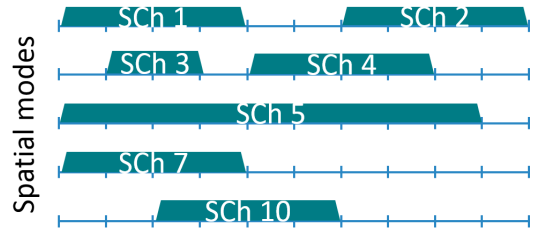

Spectral slots

(a) SDM switching scheme 1

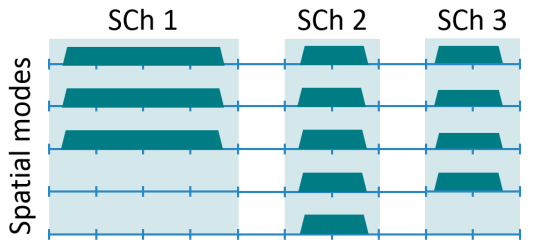

Spectral slots

(b) SDM switching scheme 2

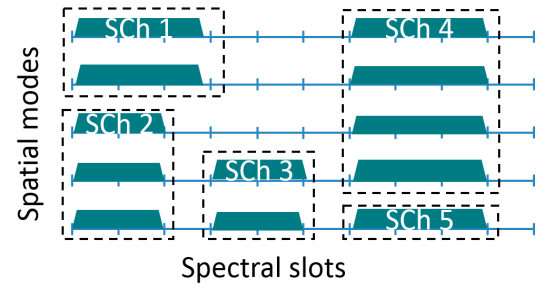

(c) SDM switching scheme 3

Figure 1: Illustration of forming superchannels in different SDM switching schemes.

a preliminary heuristic. This paper extends the work by (i) proposing mixed integer linear programming (MILP) formulations and refined heuristic algorithms for each of the SDM switching schemes in [20], and (ii) presenting simulation results based on new traffic profiles that follow the realistic flow size distribution [16]-[19].

The remainder of this paper is organized as follows. In Section II, the SDM switching schemes considered in this work are briefly introduced. The statement and objectives of the resource allocation problem are described in Section III. In Section IV, the MILPs and heuristics used to solve the problem are presented. Section V discusses numerical results. Concluding remarks are found in Section VI.

\section{SDM SWITCHING SCHEMES}

As illustrated in Fig. 2, a simple modular DC network topology [6] is studied in this paper, where the PODs are interconnected through a single optical large port count (LPC) SDM switch. Each POD is connected to the LPC switch with a single bidirectional SDM fiber. In this paper, we analyze three different SDM switching schemes proposed in previous studies [5], [6] for modular DCs [4]. Each scheme provides a certain level of network flexibility by utilizing the spatial elements offered by the SDM fiber differently.

The first scheme (A1) is referred to as uncoupled SDM with flexible-grid WDM. This architecture requries one conventional spectrum selective switch (SSS) and one spatial mux/demux per spatial element for each transmission direction of the SDM fiber [21], [22]. Flexible-grid and tunable transceivers are deployed inside the PODs to transmit/receive spectral superchannels [6]. As shown in Fig. 1(a), each spatial element in A1 operates as an independent flexible-grid WDM fiber where multiple independent spectral superchannels are established.

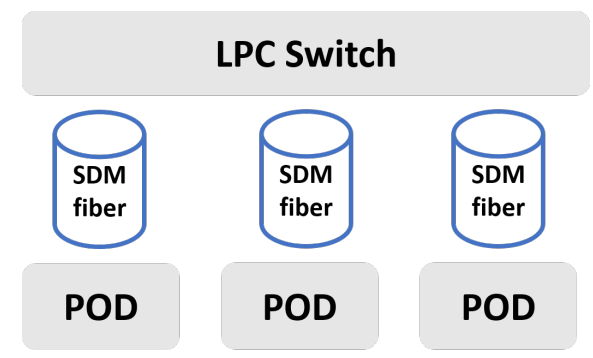

Figure 2: The modular DC network topology.
The second scheme (A2) is the coupled SDM with spectral flexibility, which is illustrated in Fig. 1(b). This architecture can be realized by equipping each POD with advance flexible-grid, tunable, MIMO transceivers to transmit/receive the spectral-spatial superchannels [22], [23]. Two large SSSs are employed to connect the PODs to the LPC switch [6]. In this scheme, spectral superchannels are expanded to all the spatial elements to create spectral-spatial superchannels with increased capacity.

The third scheme (A3) is the coupled SDM with spectral and spatial flexibility as displayed in Fig. 1(c), where the unrestricted flexibility in both spectral and spatial domains are exploited to form flexible spectral-spatial superchannels. In this architecture, the POD is also equipped with advance flexible-grid, tunable, MIMO transceivers to transmit/receive the spectral-spatial superchannels. In addition, it requires a large spectral and spatial selective switch (SSSS) to connect each POD to the SDM fiber [6].

Furthermore, assuming uncoupled spatial elements in SDM fibers, superchannels can use different spatial elements at the input and output fiber links to the switch [3], [4], [6]. However, the spectral continuity constraint is imposed on the superchannels to obtain transparent transmission.

\section{Problem Statement And Optimization OBJECTIVES}

To solve the resource allocation problem in SDM-based modular DCs, we assign spectral and spatial resources to a set of connection requests to connect the source and destination PODs. Based on the specific SDM switching scheme, different spectral-spatial superchannels can be established. In case the required resources are not available, the request is blocked.

In modular DCs, we consider that the network interconnecting PODs presents similar properties as the core tier of conventional DCs [6], whose traffic pattern varies slowly over time, on the order of several seconds or higher [16]-[18]. Hence, we assume in our offline traffic model that the connection requests in modular DCs change periodically at a fixed time interval. At the beginning of each time interval, taking the current estimation of the connection requests as an input, the resource allocation is performed offline and the corresponding network resources are reconfigured accordingly [5], [6].

The goal of the resource allocation problem is to optimize both the number of established connections $C$ and the total throughput $B$ in the modular DC network simultaneously. 
The number of established connections $C$ is related to the blocking probability $\kappa$ as $C=(1-\kappa)|T|$, where $|T|$ is the number of connection requests. In our study, the two objectives are combined linearly as $C+\beta B / t_{\text {ave }}$, where $\beta$ is a weight factor controlling the relative priority of $B$ and $C$, while $t_{\text {ave }}$ is a normalizing factor set equal to the average data rate. This objective has two components: the number of established connections is maximized when $\beta=0$, while the throughput is optimized when $\beta$ is large enough.

Assuming that the connection requests can be categorized into $n$ traffic classes each with a data rate $\tau_{i}$ for $i \in\{1, \ldots, n\}$, the objective proposed above can be rewritten as

$$
\sum_{i=1}^{n}\left(1+\beta \tau_{i} / t_{\mathrm{ave}}\right) c_{i},
$$

where $c_{i}$ is the number of established connections in class $i$. Equation (1) is a weighted sum of the numbers of established connections from different traffic classes in the network, where each traffic class is weighted by $1+\beta \tau_{i} / t_{\text {ave }}$. By tuning the value of $\beta$, the relative importance of each traffic class in the objective function is varied accordingly. This interpretation is of practical relevance because not all the traffic in the DC is equally important [17], [18]. For example, the failure of delivering short control messages may result in severe performance degradation, whereas a large volume data transfer usually has higher delay tolerance. Therefore, by differentiating the traffic classes, the resources in modular DC networks can be allocated more efficiently.

\section{Resource Allocation Algorithms}

In this section, we first present MILP formulations for the considered SDM switching schemes and then describe heuristic algorithms to provide close-to-optimal solutions to the resource allocation problems. The parameters and variables in the formulations are defined in Table I and II, respectively. The cardinal number of a set $A$ is denoted as $|A|$.

\section{Table I: INPUT PARAMETERS}

\begin{tabular}{l|l} 
Symbol & Meaning \\
\hline \hline$P$ & the set of PODs \\
\hline$T$ & the set of connection requests \\
\hline$T^{s}$ & a subset of $T$ selected by the spatial element assignment \\
\hline$\Gamma$ & the set of spatial elements in an SDM fiber \\
\hline$\Omega$ & $\begin{array}{l}\text { the set of spectral slots per spatial element in an SDM } \\
\text { fiber }\end{array}$ \\
\hline$T_{i}$ & the set of requests connecting POD $i \in P$ \\
\hline$T_{i}^{2}$ & $T_{i}^{2}=\left\{(u, v) \mid u, v \in T_{i}, u \neq v\right\}$ for $i \in P$ \\
\hline$H_{u}$ & $\begin{array}{l}\text { the set of candidate spectral-spatial superchannels for } \\
\text { connection } u \in T \text { in A3, where each element } h \in H_{u} \\
\text { has the form of }\left(\kappa_{u h}, \lambda_{u h}\right)\end{array}$ \\
\hline$f_{u}$ & $\begin{array}{l}\text { the required number of spectral slots by connection } u \in T \\
\text { in A1 and A2 }\end{array}$ \\
\hline$\kappa_{u h}$ & $\begin{array}{l}\text { the number of spectral elements required by the candidate } \\
h \in H_{u} \text { of connection } u \in T \text { in A3 }\end{array}$ \\
\hline$\lambda_{u h}$ & $\begin{array}{l}\text { the number of spatial elements required by the candidate } \\
h \in H_{u} \text { of connection } u \in T \text { in A3 }\end{array}$ \\
\hline$t_{u}$ & the requested bit rate of connection $u \in T$ \\
\hline$t_{\text {ave }}$ & $t_{\text {ave }}=\frac{1}{|T|} \sum_{u \in T} t_{u}$ \\
\hline$\beta$ & the weight factor in the objective \\
\hline \hline
\end{tabular}

Table II: DeCISION VARIABLES

\begin{tabular}{|c|c|}
\hline Symbol & Meaning \\
\hline$x_{i u k} \in\{0,1\}$ & $\begin{array}{l}\text { equals } 1 \text { if the connection } u \in T \text { in } \mathrm{A} 1 \text { uses the } \\
\text { spatial element } k \in \Gamma \text { in the fiber connecting POD } \\
i \in P\end{array}$ \\
\hline$y_{u} \in\{0,1\}$ & equals 1 if $u \in T$ is successfully provisioned \\
\hline$w_{u} \in \mathbb{N}^{+}$ & the index of the first spectral slot assigned to $u \in T$ \\
\hline$\delta_{u v} \in\{0,1\}$ & $\begin{array}{l}\text { equals } 1 \text { if the first spectral slot assigned to } u \text { is } \\
\text { smaller than the first spectral slot assigned to } v \text { for } \\
(u, v) \in \cup_{i \in P} T_{i}^{2}\end{array}$ \\
\hline$r_{i u} \in \mathbb{N}^{+}$ & $\begin{array}{l}\text { the index of the first spatial element assigned to } u \in \\
T_{i} \text { in A3, } i \in P\end{array}$ \\
\hline$z_{u h} \in\{0,1\}$ & equals 1 if $u \in T$ in A3 selects candidate $h \in H_{u}$ \\
\hline$\gamma_{i u v} \in\{0,1\}$ & $\begin{array}{l}\text { equals } 1 \text { if } u \text { and } v \text { in A3 share any spatial element, } \\
(u, v) \in T_{i}^{2}, i \in P\end{array}$ \\
\hline$q_{\text {iuv }} \in\{0,1\}$ & $\begin{array}{l}\text { equals } 1 \text { if } u \text { and } v \text { in } \mathrm{A} 3 \text { do not share any spatial } \\
\text { element and the indexes of all the spatial elements } \\
\text { in } u \text { are smaller than those in } v,(u, v) \in T_{i}^{2}, i \in P\end{array}$ \\
\hline$p_{u v} \in\{0,1\}$ & $\begin{array}{l}\text { equals } 0 \text { if } u \text { and } v \text { in } \mathrm{A} 3 \text { are both successfully } \\
\text { established, }(u, v) \in \cup_{i \in P} T_{i}^{2}\end{array}$ \\
\hline$\kappa_{u} \in \mathbb{N}^{+}$ & $\begin{array}{l}\text { the required number of spectral elements by } u \in T \\
\text { in A3 }\end{array}$ \\
\hline$\lambda_{u} \in \mathbb{N}^{+}$ & $\begin{array}{l}\text { the required number of spatial elements by } u \in T \\
\text { in A3 }\end{array}$ \\
\hline
\end{tabular}

\section{A. Mixed Integer Linear Programming}

The objective of all the formulations is the same as presented in Section III. By writing the number of established connections and total throughput as $C=\sum_{u \in T} y_{u}$ and $B=\sum_{u \in T} t_{u} y_{u}$, respectively, the objective can be expressed as $\sum_{u \in T}\left(1+\beta t_{u} / t_{\mathrm{ave}}\right) y_{u}$. All the formulations have similar constraints related to the formation of spectral-spatial superchannels, e.g., the spectral and spatial nonoverlapping constraints, but their implementations vary due to different levels of flexibilities.

1) A1: The MILP formulation for the resource allocation in A1 is presented in (2). Constraint (2b) calculates whether or not a connection request is successfully established. Constraint (2c) specifies the value of the first spectral slot's index assigned to a connection request. Constraints (2d) and (2e) enforce spectral nonoverlapping by ensuring that each spectral slot on a certain link is allocated to at most one connection request.

$$
\begin{aligned}
& \underset{y_{u}, x_{i u k}, w_{u}, \delta_{u v}}{\operatorname{maximize}} \sum_{u \in T}\left(1+\beta t_{u} / t_{\mathrm{ave}}\right) y_{u} \\
& \text { subject to } \\
& y_{u}=\sum_{k \in \Gamma} x_{i u k} \\
& 1 \leq w_{u}+f_{u}-1 \leq|\Omega| \\
& \delta_{u v}+\delta_{v u}=1 \\
& w_{u}+f_{u}-w_{v}+|\Omega|\left(\delta_{u v}+\right. \\
& \left.x_{i u k}+x_{i v k}\right) \leq 3|\Omega| \\
& \begin{array}{l}
\forall u \in T, i \in u \\
\forall u \in T \\
\forall(u, v) \in T_{i}^{2}, \\
i \in P \\
\forall(u, v) \in T_{i}^{2}, \\
i \in P, k \in \Gamma .
\end{array}
\end{aligned}
$$

2) A2: The MILP for the resource allocation in A2 is modeled in (3). Constraint (3b) calculates whether or not a connection request is successfully established. It also imposes upper and lower bounds on the first spectral slot's index of connection request $u$. Constraint (3c) specifies the spectral 
order of two connection requests if they share a common SDM fiber. Constraint (3d) is the spectral nonoverlapping constraint.

$$
\begin{array}{ll}
\underset{y_{u}, w_{u}, \delta_{u v}}{\operatorname{maximize}} \sum_{u \in T}\left(1+\beta t_{u} / t_{\mathrm{ave}}\right) y_{u} & \\
\text { subject to } & \\
y_{u} \leq w_{u} \leq\left(|\Omega|-f_{u}+1\right) y_{u} & \forall u \in T \\
\delta_{u v}+\delta_{v u}=1 & \forall(u, v) \in T_{i}^{2}, i \in P \\
w_{u}+f_{u}-w_{v}+|\Omega|\left(\delta_{u v}+\right. & \forall(u, v) \in T_{i}^{2}, i \in P . \\
\left.y_{u}+y_{v}\right) \leq 3|\Omega| &
\end{array}
$$

3) A3: The MILP formulation for the resource allocation in A3 is formalized in (4). Since multiple spectral-spatial superchannels can be used to serve a single connection request, we use a precalculated set $H_{u}$ to present all the candidates available to a connection request $u \in T$. Each element $h \in H_{u}$ is characterized by a tuple $\left(\kappa_{u h}, \lambda_{u h}\right)$, where $\kappa_{u h}$ and $\lambda_{u h}$ are the number of spectral and spatial elements needed by a superchannel $h \in H_{u}, u \in T$.

$$
\begin{array}{ll}
\text { maximize } \sum_{u \in T}\left(1+\beta t_{u} / t_{\mathrm{ave}}\right) y_{u} & \\
\text { subject to } & \\
w_{u}+\kappa_{u}-1 \leq|\Omega| & \forall u \in T \\
r_{i u}+\lambda_{u}-1 \leq|\Gamma| & \forall u \in T, i \in u \\
\kappa_{u}=\sum_{h \in H_{u}} \kappa_{u h} z_{u h} & \forall u \in T \\
\lambda_{u}=\sum_{h \in H_{u}} \lambda_{u h} z_{u h} & \forall u \in T \\
y_{u}=\sum_{h \in H_{u}} z_{u h} & \forall(u, v) \in T_{i}^{2}, i \in P \\
\delta_{u v}+\delta_{v u}=1 & \forall(u, v) \in T_{i}^{2}, i \in P \\
1-p_{u v} \leq y_{u} & \\
1-p_{u v} \leq y_{v} & \forall(u, v) \in T_{i}^{2}, i \in P \\
p_{u v} \leq 2-y_{v}-y_{u} & \forall(u, v) \in T_{i}^{2}, i \in P \\
\gamma_{i u v}+q_{i u v}+p_{u v} \leq 1 & \\
r_{i u}-\left(r_{i v}+\lambda_{v}-1\right) & \forall(u, v) \in T_{i}^{2}, i \in P \\
\leq|\Gamma|\left(1-\gamma_{i u v}\right) & \forall(u, v) \in T_{i}^{2}, i \in P \\
r_{i v}-\left(r_{i u}+\lambda_{u}-1\right) & \\
\leq|\Gamma|\left(1-\gamma_{i u v}\right) & \\
r_{i u}+\lambda_{u}-r_{i v} & \\
\leq|\Gamma|\left(1-q_{i u v}\right) & \\
r_{i v}+\lambda_{v}-r_{i u} & \\
\leq|\Gamma|\left(q_{i u v}+\gamma_{i u v}+p_{u v}\right) & \\
w_{u}+\kappa_{u}-w_{v}+|\Omega|\left(\delta_{u v}+\right. & \\
\left.\gamma_{i u v}\right) \leq 2|\Omega| &
\end{array}
$$

Constraints (4b) and (4c) specify the first spectral and spatial element assigned to a connection request, respectively. Constraints (4d) and (4e) calculate the number of spectral and spatial element used by a connection request, respectively. Constraint (4f) calculates whether or not a connection request is successfully established. Constraint ( $4 \mathrm{~g})$ specifies the relative spectral position of two connections if they share an SDM fiber. Constraint (4h) is equivalent to $1-p_{u v}=y_{u} y_{v}$. Thus, $p_{u v}$ equals 0 if both $u$ and $v$ are successfully provisioned. In constraint (4i), given two established connections $u$ and $v$ on POD $i\left(p_{u v}=0\right)$, their relative position can only be one of three cases: (i) $u$ and $v$ do not share any spatial element and all the spatial elements' indices in $u$ are less than those in $v\left(q_{i u v}=1\right.$ and $\left.\gamma_{i u v}=0\right)$, (ii) $u$ and $v$ share some spatial element $\left(q_{i u v}=0\right.$ and $\left.\gamma_{i u v}=1\right)$, and (iii) $u$ and $v$ do not share any spatial element and all the spatial elements' indices in $u$ are larger than those in $v\left(q_{i u v}=0\right.$ and $\left.\gamma_{i u v}=0\right)$. Constraints $(4 \mathrm{j})$ and (4k) specify the relative spatial position of $u$ and $v$ in case (i). Constraints (4l) and (4m) specify the relative spatial position of $u$ and $v$ in cases (ii) and (iii). Constraint (4n) specifies the relative spectral position of $u$ and $v$ if they share any spatial element.

\section{B. Heuristics}

MILPs for resource allocation are too complex to be solved in realistic scale modular $\mathrm{DCs}^{2}$. This is due to the twodimensional degrees of freedom for selecting resources. However, as is shown by the algorithm outline in Fig. 3, we can leverage the MILP formulations to partly relieve their complexities by decomposing them into spatial and spectral assignment subproblems and solve them sequentially. First, we use the spatial element assignment (SEA) to generate $T^{s}$, a subset of promising connection requests that are likely to be provisioned, and assign spatial elements to them. Then the spectral resources are assigned by solving an ensemble of firstfits (FFs) and choosing the best solution in the ensemble.

Specifically, the SEA MILPs can be formulated as follows for each scheme.

\section{1) Al:}

$$
\begin{array}{ll}
\underset{x_{i u k}, y_{u}}{\operatorname{maximize}} \sum_{u \in T}\left(1+\beta t_{u} / t_{\mathrm{ave}}\right) y_{u} & \\
\text { subject to } & \\
y_{u}=\sum_{k \in \Gamma} x_{i u k} & \forall u \in T, i \in u \\
\sum_{u \in T_{i}} f_{u} x_{i u k} \leq|\Omega| & \forall i \in P, k \in \Gamma .
\end{array}
$$

\footnotetext{
${ }^{2}$ On a $3.4 \mathrm{GHz}$ quad-core computer with $8 \mathrm{~GB}$ RAM, the computational times of (2), (3), and (4) are approximately 1 hour, 30 minutes, and more than 24 hours, respectively.
}

Algorithm 1 The mixed FF algorithm
Input:
- A set of connection requests $T^{s}$ selected by the SEA
- Normalized weight factors $p_{i}=\frac{1+\beta \tau_{i} / t_{\mathrm{ave}}}{\sum_{i=1}^{n} 1+\beta \tau_{i} / t_{\mathrm{ave}}}$ for
each traffic class $i=1, \ldots, n$

1: Let $D$ be an empty list

2: while $T^{s}$ is not empty do

3: $\quad$ Sample a traffic class $i$ with the probability equals to $p_{i}$ for $i=1, \ldots, n$

4: Uniformly sample a connection request $u$ from the traffic class $i$ in $T^{s}$

5: $\quad$ Append $u$ to the end of $D$ and delete it from $T^{s}$

6: end while

7: Allocate spectral resources to connections in $L$ one by one

Output: The spectrum assignments of established connections, i.e., the value of $w_{u}$ for all SDM switching schemes. 


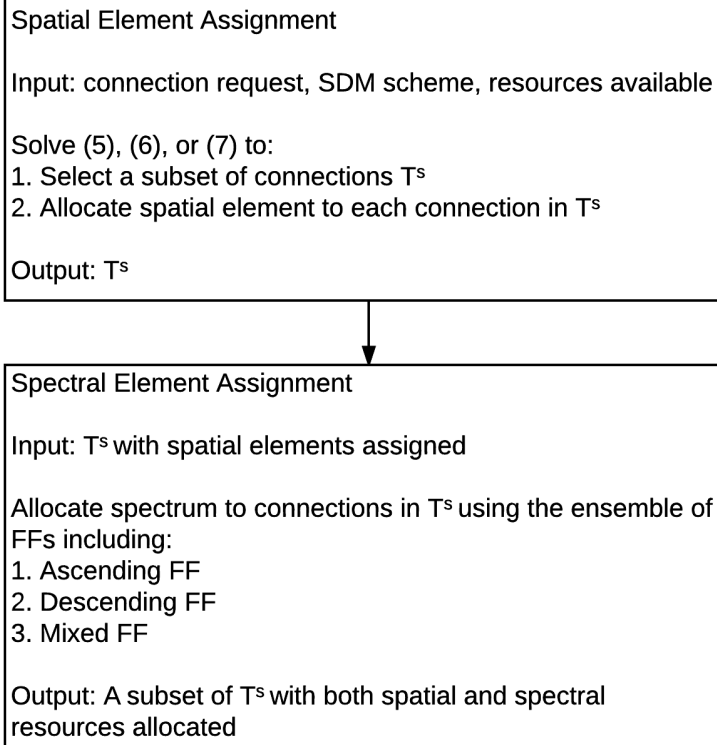

Output: A subset of Ts with both spatial and spectral resources allocated

Figure 3: Outline of the proposed heuristic algorithm.

2) $A 2$ :

$$
\begin{array}{ll}
\underset{y_{u}}{\operatorname{maximize}} \sum_{u \in T}\left(1+\beta t_{u} / t_{\mathrm{ave}}\right) y_{u} & \\
\text { subject to } & \forall i \in P . \\
\sum_{u \in T_{i}} f_{u} y_{u} \leq|\Omega| & \forall i \in{ }^{2}
\end{array}
$$

3) A3:

$$
\begin{array}{ll}
\operatorname{maximize}_{z_{u h}, \kappa_{u}, y_{u}} \sum_{u \in T}\left(1+\beta t_{u} / t_{\mathrm{ave}}\right) y_{u} & \\
\text { subject to } & \\
y_{u}=\sum_{h \in H_{u}} z_{u h} & \forall u \in T \\
\kappa_{u}=\sum_{h \in H_{u}} \kappa_{u h} z_{u h} & \forall u \in T \\
\sum_{u \in T_{i}} \kappa_{u} \leq|\Omega| & \forall i \in P .
\end{array}
$$

As can be seen from (5)-(7), the SEA subproblem is modeled by dropping all constraints related to the relative spectral position of connections in (2)-(4) and adding a constraint imposing that the capacity of each spatial element is not exceeded by the sum of the connections' capacities assigned to it. As a relaxed version of the original MILP, the SEA also calculates upper bounds of the objective functions for (2) (4). Consequently, even when it is impossible to solve (2)-(4) exactly, we can obtain an estimation of the optimal solution without heavy computational burdens.

The set of promising connection requests delivered by the SEA, i.e., $T^{s}=\left\{u \mid y_{u}=1\right\}$ in the solutions of (5)-(7), will enter the spectral element assignment stage, which is solved by an ensemble of FFs, and the final solution is the best one in the ensemble. Each of the FFs first sorts the connections according to a certain policy and then assigns spectral slots to each of them. Note that the spatial elements of each connection are already determined by the SEA and will not be changed later by the FFs.
In the ensemble of FFs, the connections are sorted by one of the following policies

i) ascending FF: ascending order of the bit rate request $t_{u}, u \in T$ [24];

ii) descending FF: descending order of the bit rate request $t_{u}, u \in T$ [25];

iii) mixed FF: a mixed order as described in Algorithm 1. Each traffic class appears in the top of the list with probabilities proportional to their relative priorities in the objective expressed in (1).

\section{NUMERICAL RESUlTS}

In this section, we first examine the performance of the proposed heuristics against the upper bounds on the objectives of the resource allocation problems given by the SEAs. We next study the relationship between the number of established connection requests and total throughput in the schemes introduced in Section II. The impact of the scheme and traffic load on the trade-off is also investigated. Finally, the trade-off is studied also under random traffic.

The dual-polarization binary phase-shift keying is assumed as the modulation format in the modular DC network. The spectrum is sliced into spectral slots of $12.5 \mathrm{GHz}$ and the number of spectral slots is linearly mapped to the provisioned capacity. A guardband of one spectral slot is assigned to each spatial element of a spectral-spatial superchannel to guarantee a satisfactory signal quality. Due to the short transmission distance inside DCs and assigned guardband, the physicallayer impairments are negligible and not considered in this paper.

The number of connection requests generated per POD is a random integer uniformly distributed in the range $\left[L_{1}(|P|-1), L_{2}(|P|-1)\right]$, where $|P|$ is the number of PODs in the modular DC and $L_{1}$ and $L_{2}$ with $0<L_{1}<L_{2}<1$ are two parameters controlling the range of the uniform distribution. Assuming that multiple flows between the same POD pair are consolidated into a single one [26], there is at most one connection request between two PODs. Two traffic classes are generated: $\tau_{1}=50 \mathrm{Gbps}$ mice flows and $\tau_{2}=400 \mathrm{Gbps}$ elephant flows. The high data rate of mice flow is because we aggregate many mice flows between two PODs into one traffic request. Two types of traffic profiles with $L_{1}=0.10, L_{2}=0.95$ and $L_{1}=0.10, L_{2}=0.35$ are used in the simulations. The $L_{1}=0.10, L_{2}=0.95$ traffic profile is used for A1 and A3, while $L_{1}=0.10, L_{2}=0.35$ is used for A2. This is because the resource utilization of A2 is much lower than those of A1 and A3 when a large amount of mice flows are present exhausting the spatial resources. Therefore, we need to adjust the traffic load for A2 to generate useful results. The value of $\beta$ is gradually varied from 0 to 10 . The elephant flows constitute $10 \%$ of the total number of flows and produce $42 \%$ of the aggregate data rate request, which follows the flow size distribution in empirical studies on DC traffic models [16]-[19]. A modular DC with $|P|=200,|\Omega|=80$, and $|\Gamma|=5$ is used in the simulations. The choice of these parameters can simplify the simulation process. Actually, the overall capacity offered by the SDM fiber is comparable 

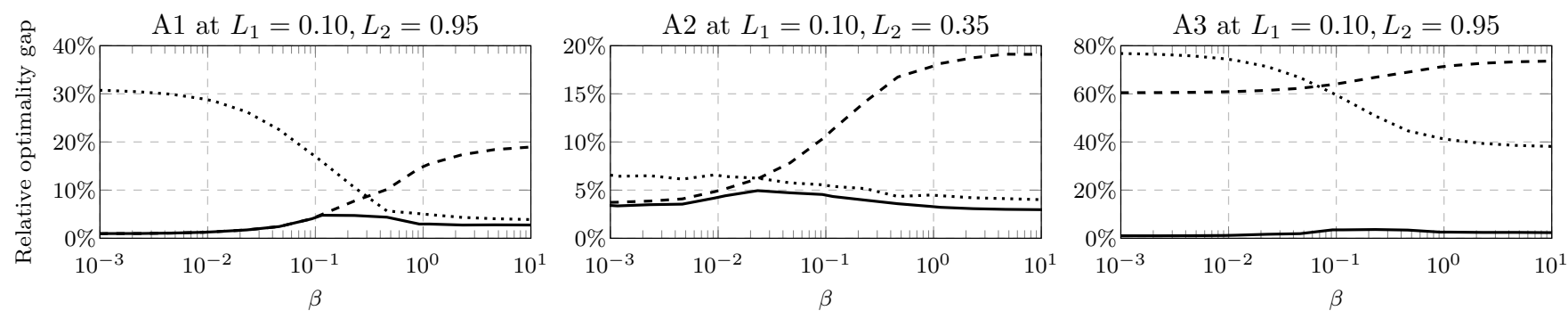

SEA+ensemble of FFs (proposed) - - - Ascending FF ...... Descending FF

Figure 4: Relative optimality gaps against the upper bounds for all the schemes.

to a WDM system utilizing full $\mathrm{C}$-band, but the cabling complexity and energy efficiency of the network is improved. Moreover, the proposed heuristic can easily scale up for larger DC networks with more spatial and spectral elements and obtain similar results since the complexity of the SEA and the ensemble of FFs are is affected by the network size. As will be shown, the trade-off between throughput and blocking probability depends only on the relative traffic load and is not related to the absolute DC size, so the selected parameters are sufficient to identify the general trade-offs in modular DCs.

\section{A. Performance of Proposed Heuristic}

The quality of the proposed heuristic algorithm can be measured by the relative optimality gap against the upper bound on the objective of the resource allocation calculated from the SEA, which is defined as

$$
g=\left(\eta_{\mathrm{ub}}-\eta_{\mathrm{he}}\right) / \eta_{\mathrm{ub}}
$$

where $\eta_{\mathrm{ub}}$ and $\eta_{\mathrm{he}}$ are the values of the objectives given by the SEA and the ensemble of FFs, respectively. The optimal solutions from the MILPs are not used in the calculation of the optimality gap because the computational complexities of MILPs are too high and cannot be solved within reasonable time. However, as will be shown below, the optimality gap is very close to zero, indicating tight upper and lower bounds estimated by the SEA and proposed heuristics, respectively.

In Fig. 4, the relative optimality gaps of the proposed heuristic are shown together with the benchmark ascending and descending FFs proposed in [6] for all the schemes. Note that the baseline FF algorithms in Fig. 4 are used for both the spatial and spectral assignments, and are different from the proposed heuristic, which assigns spatial resources by the SEA and spectral resources with the ensemble of FFs. Different traffic profiles are used for the schemes to make meaningful comparisons. As illustrated in Fig. 4, the relative optimality gaps of the proposed heuristic algorithms are close to 0 and outperform the FF benchmarks for all the schemes and all $\beta$, indicating good approximations of the optimal solutions in all cases. The gap of the benchmark ascending FF grows as $\beta$ increases, since it prioritizes mice flows and, thus, performs well only when $\beta$ is small. However, the opposite happens for the benchmark descending FF, which

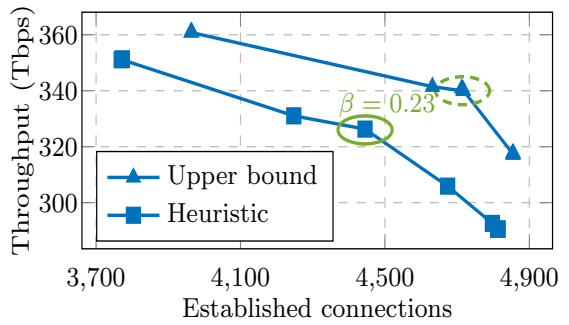

(a) A1 at $L_{1}=0.10, L_{2}=0.95$

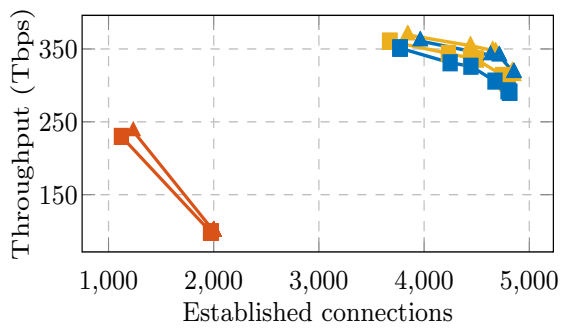

(d) All schemes at $L_{1}=0.10, L_{2}=0.95$

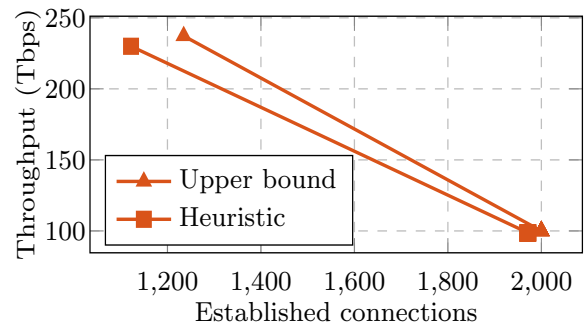

(b) $\mathrm{A} 2$ at $L_{1}=0.10, L_{2}=0.95$

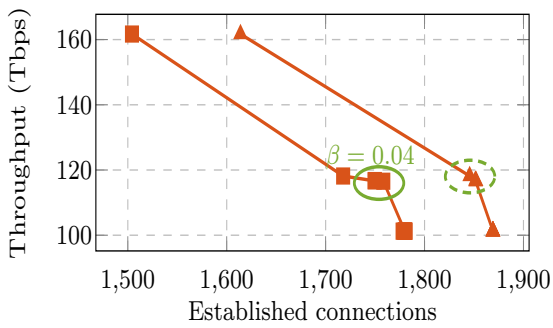

(e) A2 at $L_{1}=0.10, L_{2}=0.35$

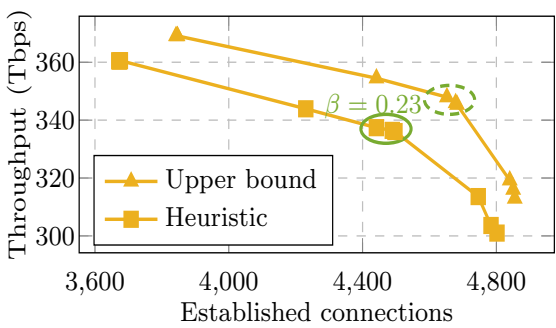

(c) A3 at $L_{1}=0.10, L_{2}=0.95$

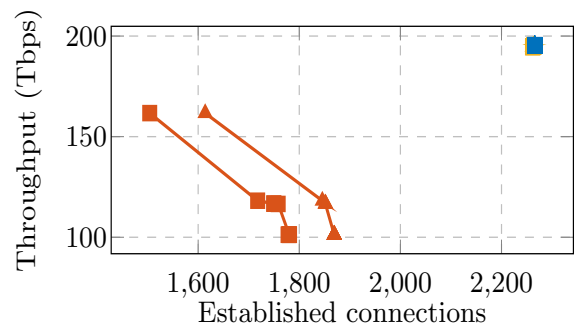

(f) All schemes at $L_{1}=0.10, L_{2}=0.35$

Figure 5: Pareto curves for different schemes when $|\Gamma|=5,|\Omega|=80,|P|=200$. The traffic load is $L_{1}=0.10, L_{2}=0.95$ in (a)-(d) and $L_{1}=0.10, L_{2}=0.35$ in (e) and (f). 
allocates elephant flows first. In A1 and A2, the gains of the proposed algorithm against the benchmarks are comparatively large for medium values of $\beta$, when the mice and elephant flows are prioritized equally. In A3, thanks to the preselection of promising connection requests performed by the SEA, the proposed algorithm demonstrates a large performance improvement compared with the FF benchmarks. We also provide the solutions from the SEA and the ensemble of FFs to the MILP solver as start points to accelerate the search for better solutions. Unfortunately, no improvement is achieved within a reasonable time, due to the high complexity of the original MILPs.

\section{B. Static Traffic Scenario}

We then investigate the relation between the throughput and number of established connections. For each SDM switching scheme, we gradually change the value of $\beta$ from 0 to 10 and solve the resource allocation problem 50 times with random traffic demands for each $\beta$. The throughput and established connections corresponding to the same $\beta$ are averaged and plotted as one point in the throughput-connection plane. Finally, the points from different $\beta$ values are connected to form a Pareto curve. The range of $\beta$ is selected such that the throughput-connection trade-off is observable, whereas either the throughput or connection will be dominant when $\beta$ is out of the chosen range.

In Fig. 5, the resulting Pareto curves for both the upper bounds on the objective of resource allocations obtained by the SEA and the proposed heuristic are shown for all the schemes. In Fig. 5(a) and 5(c), the Pareto curves of A1 and A3 bend towards the upper right corner of the throughputconnection plane. The "elbow points" correspond to $\beta=0.23$ in both schemes, which is highlighted by green ellipses. As $\beta$ increases from 0 to 0.23 , the upper bounds on the throughput increase by $8 \%$ at the expense of $3 \%$ less established connections. If $\beta$ increases above 0.23 , the numbers of connections suffer more degeneration ( $15 \%$ in the upper bounds) whereas the improvement in throughput is not significant (only $6 \%$ in the upper bounds) compared to below the "elbow points". The Pareto curves of the proposed heuristic follow a similar trend as the upper bounds. A balance between the throughput and the number of connections is achieved at the "elbow points", where both objectives are optimized without affecting each

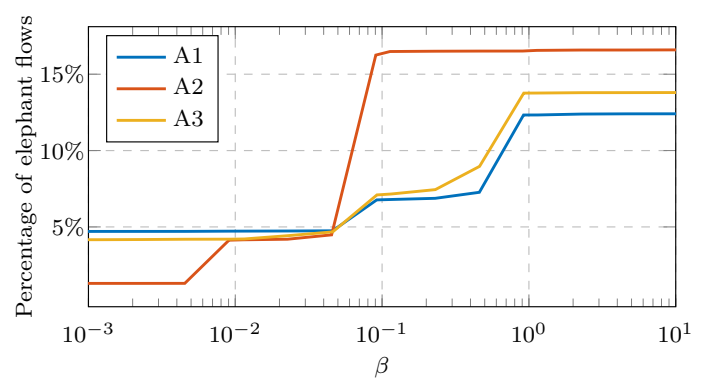

Figure 6: The fraction of the number of elephant flows in the established connections for all the schemes.

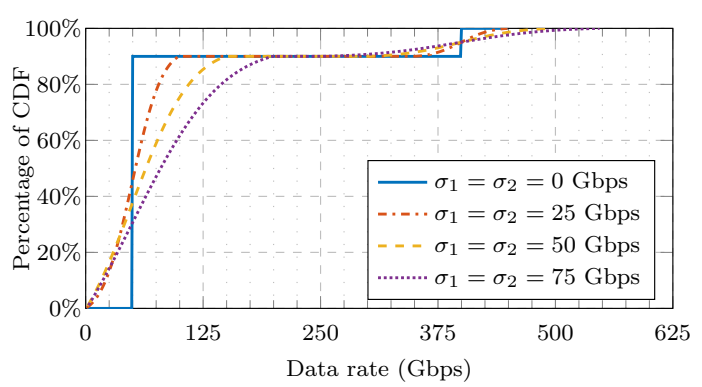

Figure 7: The CDFs of the traffic models with the same average data rates $\left(\mu_{1}=50, \mu_{2}=400 \mathrm{Gbps}\right)$ but different standard deviations.

other significantly.

In Fig. 5(b), however, we can only observe two extremes on the Pareto curves in A2. The results stay at the bottom-right corner of the figure when $\beta<0.5$ and immediately jump to the upper left corner for $\beta>0.5$. This is because A2 has much lower resource utilization compared with $\mathrm{A} 1$ and $\mathrm{A} 3$ and, thus, is over-loaded given the same connection requests as A1 and A3. When $\beta$ is small, the large number of mice flows exhaust all the resources and the elephant flows are entirely blocked. On the other hand, when $\beta$ is large enough, the elephant flows will suddenly take over and consume most of the resources, making the resource allocation problem hardly adjustable. The Pareto curve for A2 will be bent when the traffic load is light enough such that the resources are not fully dominated by only one traffic class but shared with multiple ones. This is verified by the simulation with $L_{1}=0.10, L_{2}=0.35$, as shown in Fig. 5(e), where the "elbow point" occurs at $\beta=0.04$.

The Pareto curves of all the schemes are compared in Fig. 5(d) and 5(f) for medium ( $\left.L_{1}=0.10, L_{2}=0.95\right)$ and light $\left(L_{1}=0.10, L_{2}=0.35\right)$ traffic loads, respectively. The performances of A1 and A3 are always better than A2 due to the extra flexibility provided by the SDM switching schemes. In Fig. 5(d), the Pareto curves of the upper bounds for A1 and A3 are very close to each other for all values of $\beta$, whereas A3 slightly outperforms A1 on the proposed heuristic's Pareto curves. In Fig. 5(f), A1 and A3 can provision all the connection requests, regardless of the value of $\beta$. Consequently, their Pareto curves shrink to an identical point on the throughput-

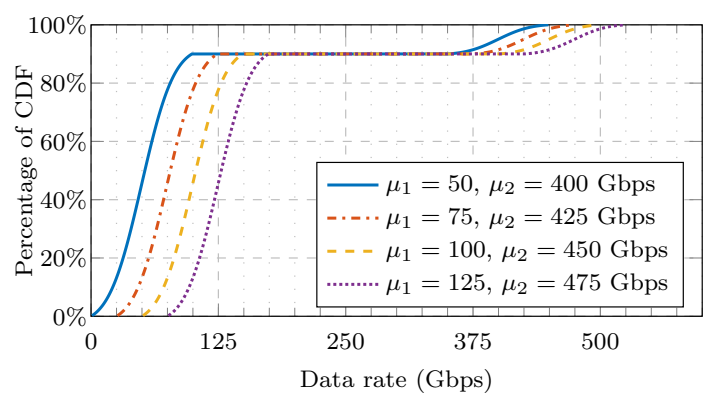

Figure 8: The CDFs of the traffic models with the same standard deviations ( $\sigma_{1}=\sigma_{2}=25 \mathrm{Gbps}$ ) but different average data rates. 


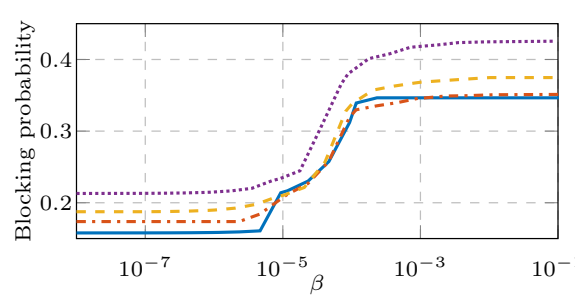

(a) The blocking probabilities of mice flows

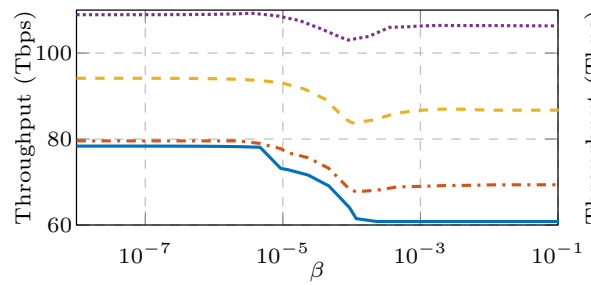

(c) The throughput of mice flows

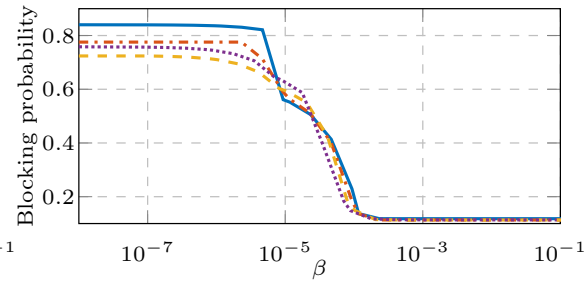

(b) The blocking probabilities of elephant flows

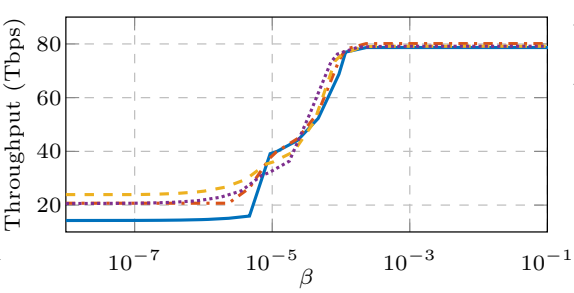

(d) The throughput of elephant flows
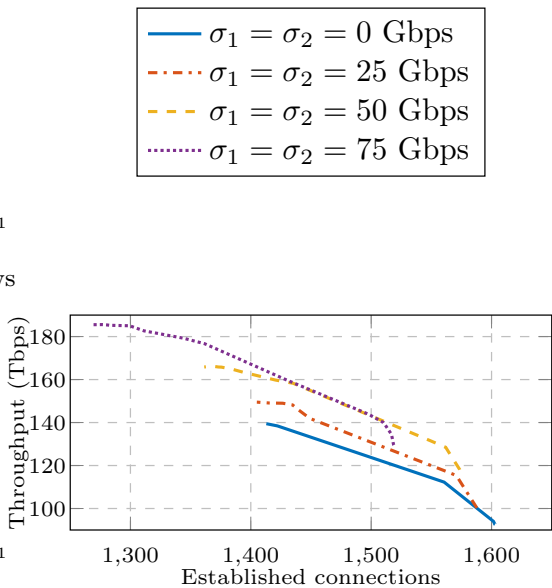

(e) The Pareto curves

Figure 9: The simulation results for A2 with the traffic models shown in Fig. 7 ( $\mu_{1}=50, \mu_{2}=400$ Gbps).

connection plane. Moreover, analogous to the performance of A2 in the light traffic scenario, the Pareto curves of A1 and A3 will become straight lines under high traffic loads. These results show that the behavior of the Pareto curve is determined by the relative traffic load and is independent of the absolute DC size.

The fraction of the number of elephant flows in the established connections is a monotonically increasing function of $\beta$ for all the schemes as shown in Fig. 6, where the elephant flows grows near the "elbow points" and finally saturate at high $\beta$. The difference between the ending and beginning of the A2 curve is larger than in A1 and A3. This is because the flexibility of allocating spectral-spatial superchannels in A2 is less than those of A1 and A3, so only relatively coarse granular resource provisioning can be achieved in A2. As a result, compared with $\mathrm{A} 1$ and $\mathrm{A} 3$, more elephant flows are blocked in $\mathrm{A} 2$ when $\beta$ is small, and more mice flows are blocked when $\beta$ is large.

\section{Stochastic Traffic Scenario}

Next, we study the impact of stochastic traffic on the tradeoff. Like in [27], we assume that the data rates of mice and elephant flows follow normal distributions $\mathcal{N}\left(\mu_{1}, \sigma_{1}\right)$ and $\mathcal{N}\left(\mu_{2}, \sigma_{2}\right)$, respectively, with negative values discarded and the distributions renormalized. Two simulation scenarios are considered. In the first scenario, the average data rates are fixed to $\mu_{1}=50$ and $\mu_{2}=400 \mathrm{Gbps}$ and the standard deviations of both flows are varied from 0 to 75 Gbps with a step size of $25 \mathrm{Gbps}$. The cumulative distribution functions (CDFs) of the chosen traffic models are shown in Fig. 7. In the second scenario, we simulate the traffic models with standard deviations fixed to $\sigma_{1}=\sigma_{2}=25 \mathrm{Gbps}$ and different average data rates. Here $\mu_{1}$ is swept from 50 to $125 \mathrm{Gbps}$ and $\mu_{2}$ from 400 to $475 \mathrm{Gbps}$, respectively, with a step size of $25 \mathrm{Gbps}$ for both flows. The CDFs of the chosen traffic models are shown in Fig. 8.

In Fig. 9, the simulation results for A2 in the first traffic scenario are illustrated. Taking the case of $\sigma_{1}=\sigma_{2}=0$ Gbps as a baseline, as the standard deviations $\sigma_{1}$ and $\sigma_{2}$ grow, the blocking probabilities of mice flows increase in Fig. 9(a),

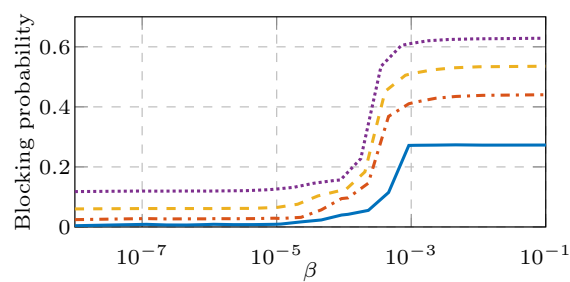

(a) The blocking probabilities of mice flows

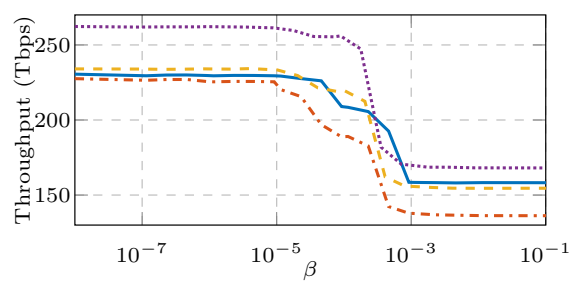

(c) The throughput of mice flows

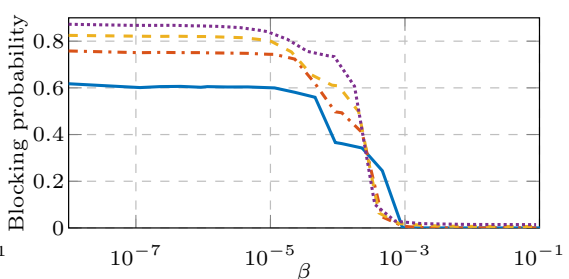

(b) The blocking probabilities of elephant flows

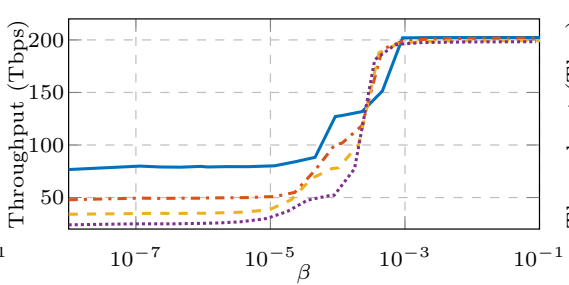

(d) The throughput of elephant flows

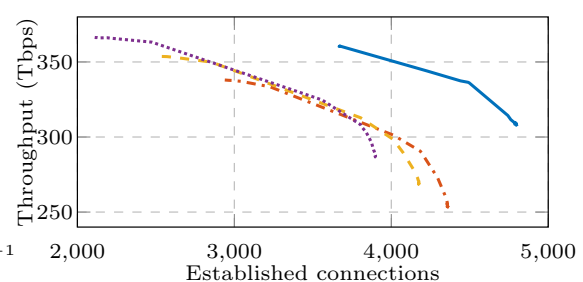

(e) The Pareto curves

Figure 10: The simulation results for A3 with the traffic models shown in Fig. 7 ( $\mu_{1}=50, \mu_{2}=400 \mathrm{Gbps}$ ). 


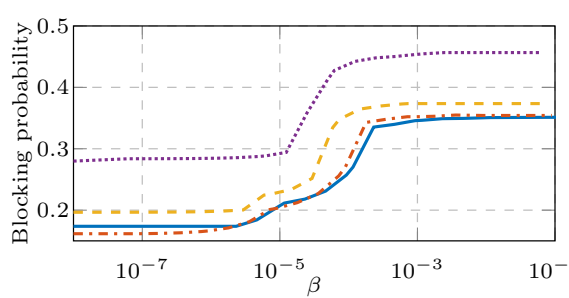

(a) The blocking probabilities of mice flows

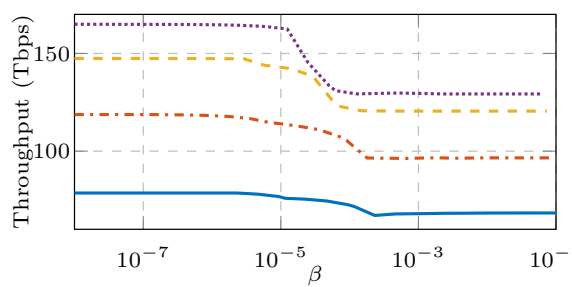

(c) The throughput of mice flows

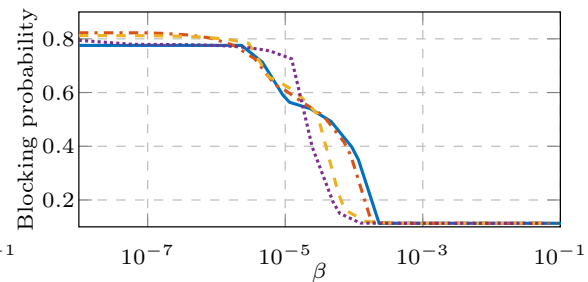

(b) The blocking probabilities of elephant flows

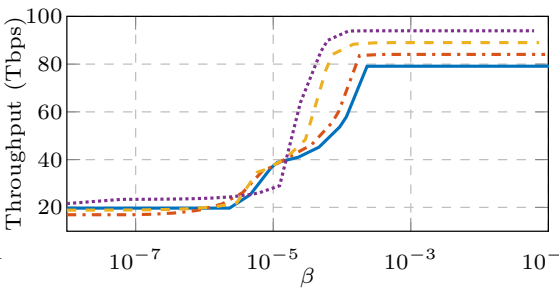

(d) The throughput of elephant flows
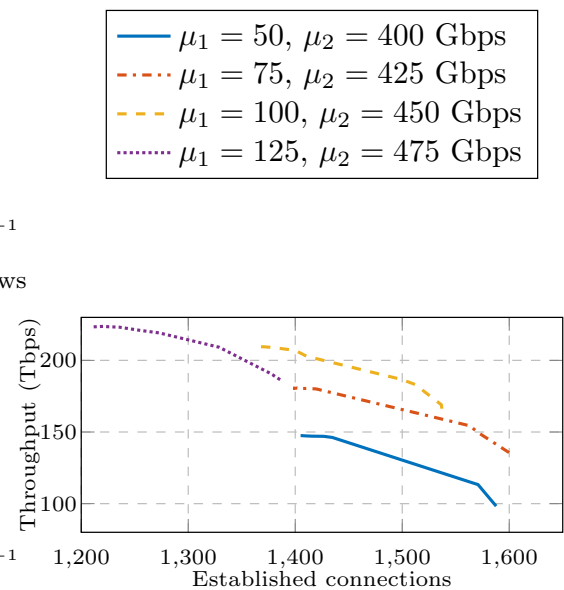

(e) The Pareto curves

Figure 11: The simulation results for A2 with the traffic models shown in Fig. 8 ( $\sigma_{1}=\sigma_{2}=25$ Gbps).

whereas those of elephant flows only fluctuate slightly in Fig. 9(b). In Fig. 9(c), the throughput of mice flows keeps increasing with an exception at $\sigma_{1}=\sigma_{2}=25 \mathrm{Gbps}$, where the throughput curve is initially very close to the baseline and has a slight increment only when $\beta$ is large. The throughput of elephant flows in Fig. 9(d) is almost unchanged relative to the baseline. In Fig. 9(e), the Pareto curves gradually move toward the upper left corner of the throughput-connection plane.

These observations are attributed to the large data rate granularity of superchannels in A2, truncated data rate distribution of mice flows, and unbalanced number of mice and elephant flows. First, the smallest superchannel in A2 utilizes one spectral element and supports a maximum data rate of $125 \mathrm{Gbps}$, which is well above the average of mice flows. Additionally, as shown in Fig. 7, even at the highest data rate variation of $75 \mathrm{Gbps}$, still more than $70 \%$ of the mice flows can fit into the smallest superchannel. These mice flows do not consume extra resources but contribute more to the throughput since their data rate distribution is truncated from below and the probability mass is shifted to higher values.
On the other hand, as the data rate variation grows, the resource usage of connection requests becomes less uniform and, thus, causes heavier resource fragmentation and higher blocking probability. However, because the number of mice flows requiring the smallest superchannel are dominant over the remaining bigger mice flows and elephant flows, the overall throughput still grows despite the rising blocking probability. The exception in Fig. 9(c) is because the distribution of mice flows is not spread out enough to be truncated when $\sigma_{1}=\sigma_{2}=25$ Gbps. Consequently, the throughput curve is the same as the baseline at smaller $\beta$ but gains a slight increment at larger $\beta$ as the proposed heuristic prefers mice flows with larger data rate to optimize the overall objective.

In Fig. 10, the simulation results for A3 in the first traffic scenario are illustrated. Taking the case of $\sigma_{1}=\sigma_{2}=0$ Gbps as a baseline, as the standard deviations $\sigma_{1}$ and $\sigma_{2}$ grow, the fragmentation becomes heavier and the blocking probabilities of both the mice and elephant flows in Fig. 10(a) and 10(b) increase. This is the reason why the Pareto curves in Fig. 10(e) are all shifted to the left and the throughput

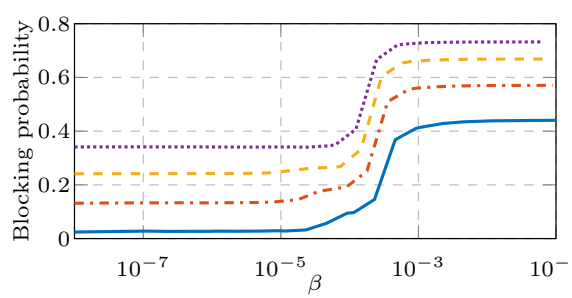

(a) The blocking probabilities of mice flows

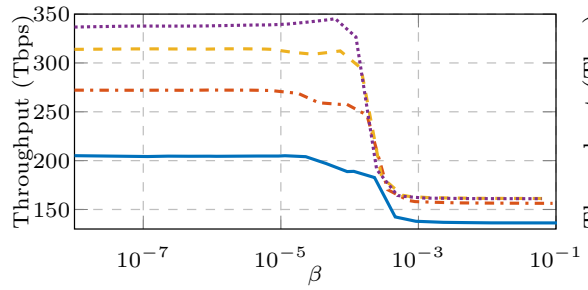

(c) The throughput of mice flows

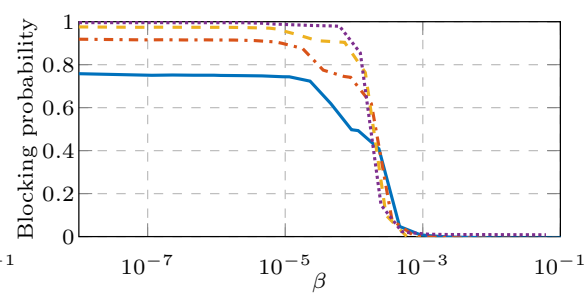

(b) The blocking probabilities of elephant flows

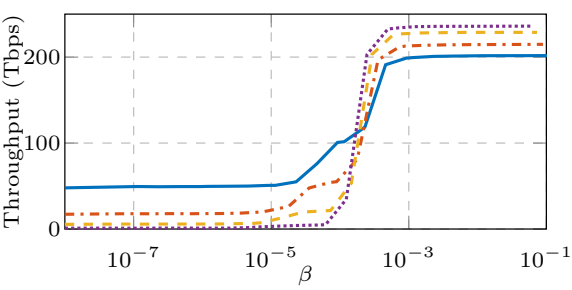

(d) The throughput of elephant flows
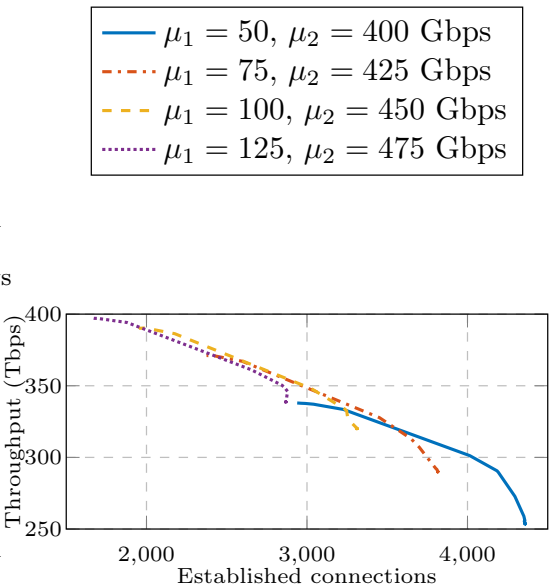

(e) The Pareto curves

Figure 12: The simulation results for A3 with the traffic models shown in Fig. 8 ( $\sigma_{1}=\sigma_{2}=25 \mathrm{Gbps}$ ). 
of the elephant flows in Fig. 10(d) drops. In Fig. 10(c), however, the throughput of mice flows behaves differently, where it first decreases at $\sigma_{1}=25 \mathrm{Gbps}$, then climbs up when $\sigma_{1}=50 \mathrm{Gbps}$, and finally becomes even higher than the baseline. When $\sigma_{1}=25 \mathrm{Gbps}$, the data rate distribution of mice flows is not spread out enough to be truncated, so the throughput of mice flows follows the reversed trend of its blocking probability. When $\sigma_{1}$ keeps growing, the distribution is truncated at zero and the average data rate of mice flow starts increasing. This effect cancels out with the reduced blocking probability at $\sigma_{1}=50 \mathrm{Gbps}$ and becomes more significant when $\sigma_{1}=75$ Gbps. Moreover, because of the dominant proportion of mice flows, the overall throughput follows the trend of mice flows and, thus, the Pareto curve first moves downward at $\sigma_{1}=\sigma_{2}=25 \mathrm{Gbps}$ and then upward.

The simulation results for A2 in the second traffic scenario are shown in Fig. 11. We consider the results of $\mu_{1}=50, \mu_{2}=$ 400 Gbps as a baseline. In Fig. 11(a), the blocking probability of mice flows stays the same at $\mu_{1}=75, \mu_{2}=425 \mathrm{Gbps}$, and then grows for larger average data rates. However, despite the fewer established mice flows, the throughput of mice flows keeps increasing in Fig. 11(c). This is because when the average data rates increases to $\mu_{1}=75, \mu_{2}=425 \mathrm{Gbps}$, the mice flows can still fit into the smallest superchannel. Hence the mice blocking probability is the same but the mice throughput increases significantly. Larger average data rates will finally diversify the resource usage and cause heavier fragmentation and, thus, induce higher blocking probability to mice flows. Meanwhile, the mice throughput increases but at a lower growth rate due to the fewer number of established mice connections. In Fig. 11(b), the blocking probability of elephant flows stays close to the baseline curve with small fluctuations, whereas the throughput of elephant flows in Fig. 11(d) tends to follow the same behavior as the mice flows but in a much slighter degree. Combining the above mentioned effects together, the resulting Pareto curve in Fig. 11(e) first shifts upward at $\mu_{1}=75, \mu_{2}=425 \mathrm{Gbps}$ and then toward the upper left corner of the throughput-connection plane.

In Fig. 12, the simulation results for A3 in the second traffic scenario are illustrated. We consider the results of $\mu_{1}=50, \mu_{2}=400 \mathrm{Gbps}$ as a baseline. As the average data rates grow, the blocking probability of mice flows in Fig. 12(a) increases as a result of heavier fragmentation. The throughput of mice flows in Fig. 12(c) also increases since the gain in average data rate outweighs the loss caused by higher blocking probability. In Fig. 12(b), the blocking probability of elephant flows grows at small $\beta$ but converges to 0 when $\beta$ is large. Consequently, the throughput of elephant flows in Fig. 12(d) decreases at small $\beta$, but the trend is reversed at large $\beta$. The resulting Pareto curve in Fig. 12(e) moves continuously toward the upper left corner of the throughput-connection plane.

The simulation results of A1 are very similar to those of A3 in both simulation scenarios and are shown in the paper. The only difference between them is that all Pareto curves of A1 are shifted slightly toward the lower left corner of the throughput-connection plane relative to their A3 counterparts. This is due to the limited switching flexibility offered by A1 in comparison with A3.
It is worth noting that the following figures of merit in DCNs are of great importance as well: i) bisection bandwidth, i.e., the minimum bandwidth available between any two equal segments of the DCN; ii) network latency, i.e., the time it takes for data to travel from its source to destination; and iii) scalability, i.e., the ability of the DCN to function well when it scales to a larger size. These key indicators are determined by both the resource allocation algorithm and DCN architecture. In this paper, due to the space limit, we mainly focus on the efficiency of the resource allocation algorithm, whereas the other figures of merit are not thoroughly investigated. Qualitatively, an efficient and balanced algorithm can achieve a fair resource utilization in the DCN and, thus, obtains a relatively high bisection bandwidth. Moreover, the computational complexity of the proposed heuristic is relatively low and is not heavily dependent on the network size. Therefore, the network latency and scalability of the DCN can also benefit from the proposed algorithm. We plan to investigate this aspect in our future work.

\section{CONCLUSIONS}

The paper presents both MILP formulations and heuristic algorithms for the resource allocation problem in three SDMbased modular DC switching schemes. The objective of the resource allocations is upper-bounded by the proposed SEA subproblem and tightly lower-bounded by the proposed heuristic algorithms. The trade-off between the number of established connections and throughput is identified. By plotting the interplay of the two objectives on the throughput-connection plane, we find that the best balance can be achieved at the "elbow point" of the Pareto curve, where both objectives are optimized simultaneously. Moreover, the shape of the Pareto curve is related to the relative traffic load and the SDM switching scheme. Despite A3's extra flexibility, it has only slightly better performance compared with A1. Whereas A2 has the lowest achievable throughput and number of established connections due to the large resource granularity of its superchannels. The trade-off persists for all the architectures in random-traffic scenario, with shifts of the Pareto curves introduced by the specific traffic distributions and resource granularity.

\section{ACKNOWLEDGMENT}

Part of this paper was presented at the Optical Communication Conference (OFC), Los Angeles, March 2017. This work was supported in part by the Swedish Research Council (VR) Grants 2012-5280 and 2014-6230.

\section{REFERENCES}

[1] J. Hamilton, "Architecture for modular data centers," arXiv preprint cs/0612110, 2006

[2] N. Farrington, G. Porter, S. Radhakrishnan, H. H. Bazzaz, V. Subramanya, Y. Fainman, G. Papen, and A. Vahdat, "Helios: a hybrid electrical/optical switch architecture for modular data centers," $A C M$ Computer Communication Review, vol. 40, no. 4, pp. 339-350, 2010.

[3] Y. Liu, H. Yuan, A. Peters, and G. Zervas, "Comparison of SDM and WDM on direct and indirect optical data center networks," in Proc. European Conference on Optical Communication (ECOC), Düsseldorf, Germany, Sept. 2016, p. M.1.F.2. 
[4] D. Klonidis, F. Cugini, O. Gerstel, M. Jinno, V. Lopez, E. Palkopoulou, M. Sekiya, D. Siracusa, G. Thouénon, and C. Betoule, "Spectrally and spatially flexible optical network planning and operations," IEEE Communications Magazine, vol. 53, no. 2, pp. 69-78, 2015.

[5] M. Fiorani, M. Tornatore, J. Chen, L. Wosinska, and B. Mukherjee, "Optical spatial division multiplexing for ultra-high-capacity modular data centers," in Proc. Optical Fiber Communication Conference (OFC), Anaheim, CA, Mar. 2016, pp. Tu2H-2.

[6] M. Fiorani, M. Tornatore, J. Chen, L. Wosinska, and B. Mukherjee, "Spatial division multiplexing for high capacity optical interconnects in modular data centers," Journal of Optical Communications and Networking, vol. 9, no. 2, pp. 143-153, 2017.

[7] D. Siracusa, F. Pederzolli, P. Khodashenas, J. Rivas-Moscoso, D. Klonidis, E. Salvadori, and I. Tomkos, "Spectral vs. spatial super-channel allocation in SDM networks under independent and joint switching paradigms," in Proc. European Conference on Optical Communication (ECOC), Valencia, Spain, Sept. 2015, p. Mo.4.6.2

[8] F. Pederzolli, D. Siracusa, J. M. Rivas-Moscoso, B. Shariati, E. Salvadori, and I. Tomkos, "Spatial group sharing for SDM optical networks with joint switching," in Proc. IEEE International Conference of Optical Network Design and Modeling (ONDM), Trento, Italy, May 2016, pp. 195-200.

[9] P. S. Khodashenas, J. M. Rivas-Moscoso, D. Siracusa, F. Pederzolli, B. Shariati, D. Klonidis, E. Salvadori, and I. Tomkos, "Comparison of spectral and spatial super-channel allocation schemes for SDM networks," IEEE Journal of Lightwave Technology, vol. 34, no. 11, pp. 2710-2716, 2016.

[10] A. Muhammad, G. Zervas, D. Simeonidou, and R. Forchheimer, "Routing, spectrum and core allocation in flexgrid SDM networks with multicore fibers," in Proc. IEEE International Conference of Optical Network Design and Modeling (ONDM), Stockholm, Sweden, May 2014, pp. 192-197.

[11] D. J. Ives, P. Bayvel, and S. J. Savory, "Routing, modulation, spectrum and launch power assignment to maximize the traffic throughput of a nonlinear optical mesh network," Photonic Network Communications, vol. 29, no. 3, pp. 244-256, 2015.

[12] D. J. Ives, P. Bayvel, and S. J. Savory, "Physical layer transmitter and routing optimization to maximize the traffic throughput of a nonlinear optical mesh network," in Proc. IEEE International Conference of Optical Network Design and Modeling (ONDM), Stockholm, Sweden, May 2014, pp. 168-173.

[13] D. J. Ives, P. Bayvel, and S. J. Savory, "Adapting transmitter power and modulation format to improve optical network performance utilizing the Gaussian noise model of nonlinear impairments," IEEE Journal of Lightwave Technology, vol. 32, no. 21, pp. 3485-3494, 2014.

[14] S. Fujii, Y. Hirota, H. Tode, and K. Murakami, "On-demand spectrum and core allocation for reducing crosstalk in multicore fibers in elastic optical networks," Journal of Optical Communications and Networking, vol. 6, no. 12, pp. 1059-1071, 2014.

[15] M. N. Dharmaweera, L. Yan, M. Karlsson, and E. Agrell, "Nonlinearimpairments- and crosstalk-aware resource allocation schemes for multicore-fiber-based flexgrid networks," in Proc. European Conference on Optical Communication (ECOC), Düsseldorf, Germany, Sept. 2016, p. Th2.P2.SC6.69.

[16] A. Greenberg, J. R. Hamilton, N. Jain, S. Kandula, C. Kim, P. Lahiri, D. A. Maltz, P. Patel, and S. Sengupta, "VL2: A scalable and flexible data center network," ACM Computer Communication Review, vol. 39, no. 4, pp. 51-62, 2009.

[17] T. Benson, A. Anand, A. Akella, and M. Zhang, "Understanding data center traffic characteristics," ACM Computer Communication Review, vol. 40, no. 1, pp. 65-72, 2010.

[18] T. Benson, A. Akella, and D. A. Maltz, "Network traffic characteristics of data centers in the wild," in Proc. Internet Measurement Conference, Melbourne, Australia, Nov. 2010, pp. 267-280.

[19] A. Roy, H. Zeng, J. Bagga, G. Porter, and A. C. Snoeren, "Inside the social network's (datacenter) network," ACM Computer Communication Review, vol. 45, no. 4, pp. 123-137, 2015.

[20] L. Yan, M. Fiorani, A. Muhammad, M. Tornatore, E. Agrell, and L. Wosinska, "Network performance trade-off in optical spatial division multiplexing data centers," in Proc. Optical Fiber Communication Conference (OFC), Los Angeles, CA, Mar. 2017, p. W3D.5.

[21] B. Shariati, J.-M. Rivas-Moscoso, D. Marom, S. Ben-Ezra, D. Klonidis, L. Velasco, and I. Tomkos, "Impact of spatial and spectral granularity on the performance of SDM networks based on spatial superchannel switching," IEEE Journal of Lightwave Technology, vol. 35, no. 13, pp. 2559-2568, 2017.
[22] D. M. Marom, P. D. Colbourne, A. Derrico, N. K. Fontaine, Y. Ikuma, R. Proietti, L. Zong, J. M. Rivas-Moscoso, and I. Tomkos, "Survey of photonic switching architectures and technologies in support of spatially and spectrally flexible optical networking," Journal of Optical Communications and Networking, vol. 9, no. 1, pp. 1-26, 2017.

[23] D. M. Marom and M. Blau, "Switching solutions for WDM-SDM optical networks," IEEE Communications Magazine, vol. 53, no. 2, pp. 60-68, 2015.

[24] B. C. Chatterjee, N. Sarma, and E. Oki, "Routing and spectrum allocation in elastic optical networks: a tutorial," IEEE Communications Surveys \& Tutorials, vol. 17, no. 3, pp. 1776-1800, 2015.

[25] J. Zhao, H. Wymeersch, and E. Agrell, "Nonlinear impairment-aware static resource allocation in elastic optical networks," IEEE Journal of Lightwave Technology, vol. 33, no. 22, pp. 4554-4564, 2015.

[26] H. Rastegarfar, M. Glick, N. Viljoen, M. Yang, J. Wissinger, L. LaComb, and N. Peyghambarian, "TCP flow classification and bandwidth aggregation in optically interconnected data center networks," Journal of Optical Communications and Networking, vol. 8, no. 10, pp. 777-786, 2016.

[27] B. Shariati, D. Klonidis, D. Siracusa, F. Pederzolli, J. Rivas-Moscoso, L. Velasco, and I. Tomkos, "Impact of traffic profile on the performance of spatial superchannel switching in SDM networks," in Proc. European Conference on Optical Communication (ECOC), Valencia, Spain, Sept. 2016, p. M.1.F.1. 